\title{
Collective behaviour in cascade model depends on turn of the choice
}

\author{
Saori Iwanaga* \\ Department of Maritime Safety Technology, \\ Japan Coast Guard Academy, \\ 5-1 Wakaba, Kure, Hiroshima, 737-8512, Japan \\ Email: s-iwanaga@jcga.ac.jp \\ *Corresponding author
}

\author{
Akira Namatame \\ Department of Computer Science, \\ National Defense Academy, \\ 1-10-20 Hashirimizu, Yokosuka, \\ Kanagawa, 239-8686, Japan \\ Email: nama@nda.ac.jp
}

\begin{abstract}
There are growing interests for studying collective behaviour including the dynamics of markets, the emergence of social norms and conventions, and collective phenomena in daily life such as traffic congestion. In cascade model, collective behaviour is affected in the structure of the social network and threshold to make decision, and the collective behaviour was stochastic, similar to threshold model in local interaction. In both model, although decisions of agents are deterministic, collective behaviour of population become stochastic. In previous work, we analysed origin of stochastic collective behaviour and we found that collective behaviour depends on the initial behaviour of high degree in threshold model in local interaction. In this paper, we analysed origin of stochastic collective behaviour in cascade model. We analysed why collective behaviour is stochastic in scale free network. Then, we show that turn of the choice effects on collective behaviour not the first choices.
\end{abstract}

Keywords: collective behaviour; agent; social network; network degree; threshold model; multi agent simulation; cascade model; choice.

Reference to this paper should be made as follows: Iwanaga, S. and Namatame, A. (2016) 'Collective behaviour in cascade model depends on turn of the choice', Int. J. Automation and Logistics, Vol. 2, Nos. 1/2, pp.93-107.

Biographical notes: Saori Iwanaga is a Professor of Department of Maritime Safety Technology at Japan Coast Guard Academy (JCGA) since 2012. She received her BE in Applied Chemistry Engineering from Utsunomiya University, Japan. She received her MS and $\mathrm{PhD}$ degrees in Computer Science from National Defense Academy, Japan. She has worked at JCGA since 2006. She is interested in complex theory, evolutionary games. She is a member of Information Processing Society of Japan and Japan Society for Safety Engineering. 
Akira Namatame is a Professor of Department of Computer Science National Defense Academy of Japan. He holds a degree of Engineering in Applied Physics from National Defense Academy. He holds a Master of Science in Operations Research and a $\mathrm{PhD}$ in Engineering-Economic System from Stanford University. His research interests include multi-agents, game theory, evolution and learning, complex networks, economic sciences with interaction agents and a science of collectives. He is a member of the AAAI, IEEE, and SICE.

This paper is a revised and expanded version of a paper entitled 'Collective behavior in cascade model depend on turn of choice' presented at the 18th Asia Pacific Symposium on Intelligent and Evolutionary Systems, Singapore, 10-12 November 2014.

\section{Introduction}

There are growing interests for studying collective behaviour including the dynamics of markets, the emergence of social norms and conventions, and collective phenomena in daily life such as traffic congestion. Many researchers have pointed out that an equilibrium analysis does not resolve the question of how agents behave in a particular interdependent decision situation. Threshold model (Schelling, 1978) has been postulated as one explanation for the contagion. It is often argued, it is hard to see what can advance the discussion short of assembling a collection of agent, putting them in the situation of interest, and observing what they do (Huberman and Glance, 1993).

In examining collective behaviour, we shall draw heavily on the interactions of agents. We also need to work on two different levels: the microscopic level, where the decisions of the agent agents occur, and the macroscopic level where collective behaviour can be observed (Sipper, 1997). The greatest promise lies in analysis of linking microscopic behaviour to macroscopic behaviour (Schweitzer, 2002). What makes collective behaviour interesting and difficult is that the entire aggregate outcome is what has to be evaluated, not merely, how each person does within the constraints of her own environment? The performance of the collective system depends crucially on the type of interaction as well as the heterogeneity in preference of agents (Kirman, 1997).

Feng et al. (2012) brings together agent-based models and stochastic models of complex systems in financial markets and show how agent decisions give rise to macroscopic actions. Additionally, the heterogeneity in agents' investment horizons gives rise to long-term memory in volatility. Using market data, Kenett et al. (2012) provides new information about the uniformity present in the world's economies. From their analysis, it becomes evident that this uniformity does not only stem from an increase of correlation between markets, but that there has also been an ongoing simultaneous shift towards uniformity in each single market.

There are many situations where interacting agents can benefit from coordinating their behaviour. Coordination usually implies that increased effort by some agents leads the remaining agents to follow suit, which gives rise to multiplier effects. Examples where coordination is important include trade alliance, the choice of compatible technologies or conventions such as the choice of a software or language. These situations can be modelled as coordination games in which agents are expected to select 
the strategy the majority do (Schelling, 1978). The traditional game theory, however, is silent on how agents know which equilibrium should be realised if a coordination game has multiple equally plausible equilibria, where these can be Pareto ranked (Arthur, 1994). This silence is more surprising in games with common interest since one expects that agents will coordinate on the Pareto dominant equilibrium (Hansarnyi and Selten, 1988). The game theory has been also unsuccessful in explaining how agents should behave in order to improve an equilibrium situation (Fudenberg and Levine, 1998).

Often an agent's decision depends on the decisions of others because they have limited information about the problem or limited ability to process the information (Shelling, 1978; Rubinstein, 1998). An agent's payoff is a function of the actions of others (Huberman et al., 1993; Fudenberg and Levine, 1998). In particular, in the diffusion of a new technology (Arthur, 1989), early adopters impose externalities on later ones by rationally choosing technologies to suit only themselves. Then, agent has an incentive to pay attention to the decisions of others. This is known as binary decisions with externalities (Schelling, 1978).

Threshold model (Schelling, 1978) has been postulated as one explanation for the contagion. Schelling shows by example of attendance the optional Saturday morning review session. For people, attendance depends on the percentage of attendance. The critical point which the benefit exceeds the cost of attendance is threshold. Schelling assumes that all people know the others' decision and deals with stability of equilibrium of several threshold distributions. And in threshold model, we (Iwanaga and Namatame, 2012) showed that collective behaviour is affected in the structure of the social network, the initial collective behaviour and diversity of threshold. Moreover, we show the effect of number of interaction on collective behaviour. Then, in threshold model, we found that collective behaviour depends on the initial behaviour of high degree agents in several social networks, such as random network, small world network or scale free network (Iwanaga and Namatame, 2013).

Contagion is said to occur if one behaviour can spread from a finite set of agents to the whole population. When can behaviour that is initially adopted by only an infinite set of agents spread to the whole population? Morris (2000) shows that maximal contagion occurs when local interaction is sufficiently uniform and there is low neighbour growth, i.e., the number of agents who can be reached in $k$ steps does not grow exponentially in $k$. López-Pintado (2006) showed that there exists a threshold for the degree of risk dominance of an action such that below the threshold, contagion of the action occurs. He also showed that networks with intermediate variance (where the connectivity of the lowest connectivity nodes are not so low) are best for diffusion purposes. Meanwhile, Watts (2002) showed that when the network of interpersonal influences is sufficiently sparse, the propagation of cascades is limited by the global connectivity of the network; and when it is sufficiently dense, cascade propagation is limited by the stability of the agent nodes. Therefore, the rate at which a social innovation spread depends on three factors: the topology of network, the payoff gain of the innovation and the amount of noise in the best response processes (Young, 2011). Montanari and Saberi (2010) shows that innovation spreads much more slowly on well-connected network structure dominated by long range links than in low dimensional ones dominated and Komatsu and Namatame (2012) obtains the optimal network for good cascade using genetic algorithm and they show the network have a sufficient number of vulnerable nodes and hub node of medium size. We (Iwanaga and Namatame, 2013) compared threshold model with 
cascade model and showed that collective behaviour is affected in the structure of the social network and threshold to make decision and the collective behaviour was stochastic. Moreover, collective behaviour is almost same as threshold model, though the decision is not interactive and simultaneously. Then, collective behaviour in threshold model is similar to cascade model.

To illustrate how important spatial structure is to the emergence of cooperation in society, Nowak (2006) and Axelrod (1984) have investigated lattice models of agents confronted with a social dilemma. At the other extreme, most of human social networks were regarded as random networks whose nodes are connected randomly because of its large scale and complexity. In reality, Albert and Barabási (2000) found that many complex networks have a scale free structure. Moreover, another kind of network structure, small world, has been researched (Watts, 1999). Of course, the number of agents is large and the relationship is assumed complex. However, the world is much smaller than we think. Real-world networks have a small average shortest path length that defined as the average number of steps along the shortest paths for all pairs of agents.

On the other hand, Hasan and Ukkusuri (2011) deal with a threshold model of social contagion originally proposed in network science literature. In addition, they show that faster propagation of warning is observed in community networks with greater inter community connections.

We deal with collective behaviour in several social networks. Collective behaviour is affected in the structure of the social network and threshold to make decision, and the collective behaviour was stochastic in threshold model and cascade model. Although decisions of agents are deterministic, collective behaviour of population becomes stochastic. In previous work, we analysed origin of stochastic collective behaviour and we found that collective behaviour depends on the initial behaviour of high degree in threshold model and local interaction. In this paper, we analysed origin of stochastic collective behaviour in cascade model. We analysed why collective behaviour is stochastic in scale free network. We focus on the effect of network degree. Then, we show that turn of the choice effects on collective behaviour not the first choices.

\section{Model}

We consider the following dynamics to describe the evolution of agents' choices through time step. At time step $t$, each agent plays a $2 \times 2$ game with a neighbour and chooses an action from the space $\mathrm{S}=\{\mathrm{S} 1, \mathrm{~S} 2\}$ (López-Pintado, 2006; Watts, 2002). The assumption that an agent cannot make his action contingent on his neighbour's action is natural in this context. Otherwise, the behaviour of an agent would be independent of social network. Payoffs from each interaction in each period are given by a function (s, s'), where $\mathrm{s}, \mathrm{s}^{\prime} \in \mathrm{S}$ and they are summarised in the following symmetric matrix as shown in Table 1, where $0 \leq \theta_{i} \leq 1$. In this matrix, if agent Ai chooses same strategy as the other agent, he can get the positive payoff $1-\theta_{i}$ or $\theta_{i}$, otherwise he receives nothing. This means coordination game. This is also called as conformity model. This payoff matrix can be translated from Stag Hunt game. Nash equilibria of the payoff matrix are (S1, S1) and (S2, S2). Agent Ai's payoff from playing $s_{i} \in\{\mathrm{S} 1, \mathrm{~S} 2\}$ when the strategy profile of the remaining agents s' is given by $\prod\left(s_{i}, s_{-i}\right)=\sum_{j \in N_{i}} \pi\left(s_{i}, s_{j}\right)$. Thus, an agent's payoff is simply the sum of the payoffs obtained across all the bilateral games in which he is 
involved. Agents select the action that maximises his benefits given the action of others in the previous time step (a myopic best response).

Here we define $p_{i}(t)$ as the proportion of agent Ai's neighbours who choose $\mathrm{S} 1$ at time step $t$. Figure 1 explain the social network and the proportion of agent's neighbours who choose $\mathrm{S} 1$ at time step $t$. A node means an agent and links mean neighbouring social network. Each agent has theta $\theta_{i}$ and can decide the choice S1 or S2 at given time step according to the proportion of neighbours who choose $\mathrm{S} 1$, which is $p_{i}(t)$. Because each agent has idiosyncratic theta $\theta_{i}$ and the proportion of neighbours who choose $\mathrm{S} 1$, which is $p_{i}(t)$, is different each other, the decision at next time step is deferent each other.

Table 1 Payoff matrix of agent Ai $\left(0 \leq \theta_{i} \leq 1\right)$

\begin{tabular}{lcc}
\hline \multirow{2}{*}{ Choice of agent Ai } & \multicolumn{3}{c}{ Choice of other agents } \\
\cline { 2 - 3 } & $S 1$ & $S 2$ \\
\hline S1 & $1-\theta_{i}$ & 0 \\
S2 & 0 & $\theta_{i}$ \\
\hline
\end{tabular}

Figure 1 Social network and the proportion of agent's neighbours who choose S1 at time step $t$

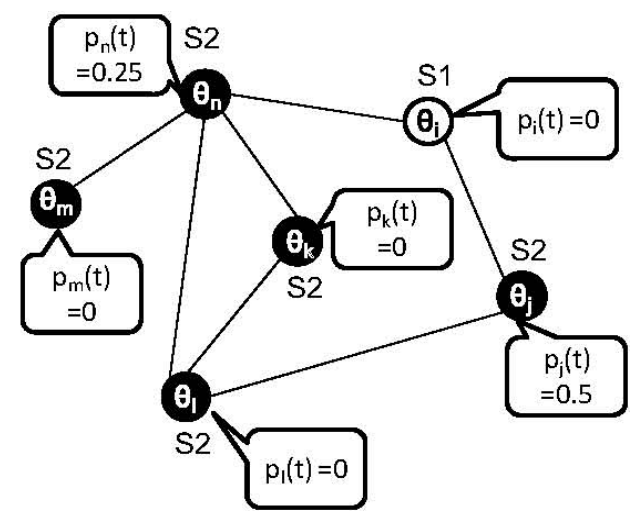

Note: Agent, links: neighbouring social network, choice: S1 or S2.

If the proportion of neighbours $p_{i}(t)$ is higher than or equal to theta $\theta_{i}$, then agent Ai's best response is to choose $\mathrm{S} 1$. Otherwise, Ai chooses S2. The value of theta $\theta_{i}$, namely the degree of risk dominance of action S1, specifies a lower bound for the fraction of agents that must be choosing S1 in order to make action S1 preferred to action S2. If $\theta_{i}<1 / 2$, action $\mathrm{S} 1$ is risk dominant. In addition, the more risk dominant action $\mathrm{S} 1$ is the lower the value of $\theta_{i}$. This rule is given by these functions.

$$
\begin{aligned}
& p_{i}(t) \geq \theta_{i}: \text { Agent Ai chooses S1 } \\
& p_{i}(t)<\theta_{i}: \text { Agent Ai chooses S2 }
\end{aligned}
$$

For example, if $\theta_{i}$ of all agents are 0.1, agent $\mathrm{An}$ and $\mathrm{Aj}$ in Figure 1 choose $\mathrm{S} 1$ at next time step. Other agents choose $\mathrm{S} 2$ at next time step.

The model differs from cascade models that Watts or López-Pintado deals with in some respects. All these features; simultaneity, interactive interaction and network heterogeneity are essential to collective behaviour. 
1 Interactive interaction: Each agent can revise his behaviour both of two alternatives, that is the decision is two ways. In original threshold model, an agent can change fromS1 to S2 or from S2 to S1. However, in cascade model, once an agent has switched on one alternative $\mathrm{S} 1$, it remains on $\mathrm{S} 1$ for the duration of the dynamics.

2 Simultaneity: In cascade model, a certain probability agents are chosen each period to revise their strategy. While, each agent decides his behaviour depend on the neighbours' behaviour in previous time step for each time step, which is simultaneously.

3 Heterogeneous networks: typically modelled on regular lattices, here we are concerned with heterogeneous networks, networks in which agents have different numbers of neighbours.

\section{Cascade model}

\subsection{Settings}

At first, we defused the interactive interaction and focus on the effect of two-way interaction. That is, agent can only change the behaviour for S1 and the decision rule is given by this function, which is same as function (1).

$$
p_{i}(t) \geq \theta_{i}: \text { Agent Ai chooses S1 }
$$

For example, if $\theta$ of all agents are 0.1 , agent $\mathrm{An}$ and $\mathrm{Aj}$ in Figure 1 choose $\mathrm{S} 1$ at next time step. Agent Ai continue to choose S1at next time step, because agent cannot change to S2. Other agents choose S2 at next time step.

Next, we defused the simultaneity and focus on the effect of simultaneity. That is, randomly chosen an agent can only decide at the same time step.

At last, we set four models of social network for each population, regular network, small world network, random network and scale free network, which Kawachi algorism from regular networks, other three types of networks emerged (Kawachi et al., 2004). At first, we made regular in which there are network 1,000 agents with ten links, and arrange the network for small world network, random network and scale free network using Kawachi algorism Then, their average degrees are 10. Kawachi et al. proposed generation algorithm from regular network to four networks by each agent's with a link of the same number changing a link. That is, a node whose number of links is large must be much larger and a node whose number of links is small must be much smaller. When all links of each node have been considered once, the procedure is repeated several time steps. For scale free network, we set probability $\alpha$ and times $\gamma$ as shown in Table 2. With set probability $\alpha$, link between agent $\mathrm{Ai}$ and Agent $j$ is deleted. Then, if degree of agent $\mathrm{Ai}$ is greater than degree of agent $\mathrm{Aj}$, agent $\mathrm{Ai}$ rewired to another agent. Otherwise, agent $\mathrm{Aj}$ rewired to another agent. When all links of each node have been considered once, the procedure is repeated and times $\gamma$.

We show the social networks by two parameter, clustering coefficient and characteristic path length. Clustering coefficient $C$ is the extent to which nodes adjacent to any node are linked to each other. Characteristic path length $L$ is the median of the means of the shortest path lengths connecting each node to all other nodes. 
The clustering coefficient $C_{i}$ of $\Gamma i$ characterises the extent to which nodes adjacent to nodes $A_{i}$ are linked to each other. Where the neighbourhood $\Gamma_{i}$ of a node $A_{i}$ is the subgraph that consists of the nodes linked to $A_{i}$ (not including $A_{i}$ itself). More precisely, $C_{i}=\left|E\left(\Gamma_{i}\right)\right| /\left(\begin{array}{c}k_{i} \\ 2\end{array}\right)$ where $\left|E\left(\Gamma_{i}\right)\right|$ is number of edges in the neighbourhood of $A_{i}$ and $\left(\begin{array}{c}k_{i} \\ 2\end{array}\right)$ is the total number of possible edges in $\Gamma_{i}$. Then, the clustering coefficient $\mathrm{C}$ of $\mathrm{G}$ is averaged $\mathrm{C}=C_{i}$ over all $A_{i} \in \mathrm{V}(\mathrm{G})$.

The characteristic path length $\mathrm{L}$ of a graph $\mathrm{G}$ is the median of the means of the shortest path lengths connecting each node $A_{i} \in \mathrm{V}(\mathrm{G})$ to all other nodes, where $\mathrm{V}(\mathrm{G})$ is the set of nodes of the graph G. In other words, first, we calculate $\mathrm{d}(\mathrm{i}, \mathrm{j}) \vee A_{i} \in \mathrm{V}(\mathrm{G})$, and then we find $\bar{d}_{i}$ for each $A_{i}$. Finally, we define $\mathrm{L}$ as the median of $\bar{d}_{i}$.

We calculate the standardised clustering coefficient and the standardised characteristic path length. We also show the standard clustering coefficient and standardised characteristic path length in Table 2. C0 means the clustering coefficient of regular network and the value is 0.67 . L0 means the characteristic path length of regular network and the value is 50.45. The characteristic path length of scale free network is infinite, because there are closed subnetwork that do not connect the other nodes at all. Therefore, the standardised characteristic path length of scale free network is infinite.

Table 2 Probability, times, clustering coefficient and characteristic path length

\begin{tabular}{lcccc}
\hline & Regular & $\begin{array}{c}\text { Small } \\
\text { world }\end{array}$ & Random & $\begin{array}{c}\text { Scale } \\
\text { free }\end{array}$ \\
\hline Probability & 0 & 0.01 & 0.5 & 1.0 \\
Times $\gamma$ & 0 & 3 & 6 & 20 \\
Standardised clustering coefficient C/C0 & 1.00 & 0.99 & 0.15 & 0.11 \\
Standardised characteristic path length L/L0 & 1.00 & 0.21 & 0.065 & $\infty$ \\
\hline
\end{tabular}

The scale free network is organised as shown in Figure 2, which is shown by log-log graph.

Figure 2 The degree distribution of scale free network

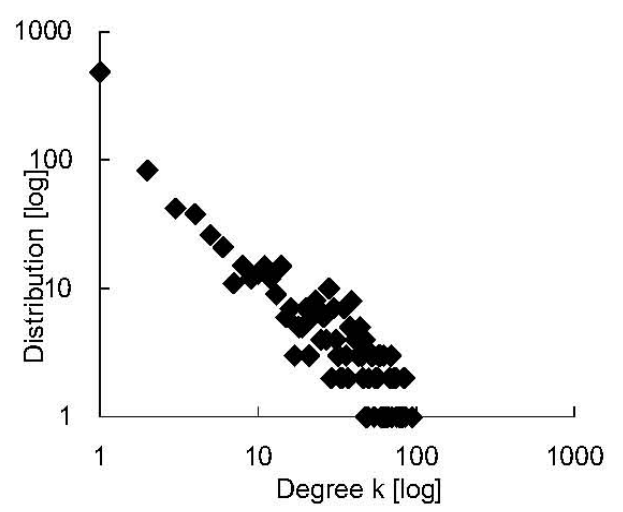

We set that all agents have same payoff matrix and theta $\theta_{i}=\theta$ of all agents as 0.1 . Then, considering pair of agents, Nash equilibrium is for both of them to choose $\mathrm{S} 1$ or for both 
of them to choose S2. Therefore, for population, Nash equilibrium is for all agents to choose $\mathrm{S} 1$ or to choose $\mathrm{S} 2$.

\subsection{Results}

We denote the collective behaviour $p(t)$ that the proportion of agents having chosen $\mathrm{S} 1$ in whole population at time step $t$. Here, we set the initial collective behaviour as 0.01 , which means only $1 \%$ of agents choose S1 at first time step. Watts (2002) sets that a single node chooses $\mathrm{S} 1$ at first time step in 10,000 nodes and simulates. If there are not node chooses S1 at all at first, choice of S1 does not spread according to function (1). A single node or small nodes need for cascade or contagion. We assume that all agents choose at random at first time step. Each agent gets uniform random number between 0 and 99 at first step. If the random number is equal to zero, the agent chooses S1 at first time step. Otherwise, agent chooses S1 at first step. Then, each agent makes decision depend on rule of cascade model given by function (1) each time step, and then collective behaviour turns.

Figure 3 shows the simulation results in scale free network, where theta $\theta$ is 0.1 . When initial collective behaviour is 0.01 , final collective behaviours depends on trials. Final collective behaviour sometime converges to 0.01 and $1 \%$ of agents choose S1 at last. Because agent cannot change for S2, there few agents who choose S1 at first time step remain at last time step. However, other time, final collective behaviour converges to 1.0 and all agents choose $\mathrm{S} 1$ at last. In latter case, there occur contagion and triggered by small proportion of S1 spread to the whole population, which is rare case.

Figure 3 The transition of collective behaviour in scale free network

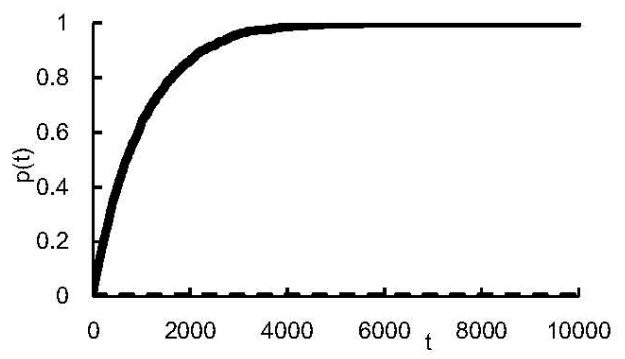

We set theta $\theta$ at intervals of 0.1 from 0.0 to 1.0. We simulate until 10,000 time step, we call this as a trial. Then, we simulate 100 trials per each theta $\theta$ and investigate the final collective behaviour. Figure 4(a) shows the simulation results in scale free network. The $\mathrm{x}$-axis represents theta $\theta$ and the $\mathrm{y}$-axis represents the final proportion of agents who choose $\mathrm{S} 1$, which we define final collective behaviour as $p^{*} .100$ trials are plotted for each theta $\theta$. The collective behaviour depends on theta $\theta$ and becomes 0.01 or 1.0 , stochastically.

If theta is greater or than equal to 0.6 , collective behaviour converges to 0.01 and almost agents choose $\mathrm{S} 2$ at last in any trials. Otherwise, theta is 0.0 , collective behaviour converges to 1.0 and all agents choose S1 at last. However, if theta is between 0.1 and 0.5 , collective behaviours become 0.01 or 1.0 depending on the trials. We found that collective behaviour is stochastic. 
Other viewpoints of results are shown in Figure 4(b). In Figure 4(b), the x-axis represents theta and the $y$-axis represents the histogram of the final collective behaviour $\mathrm{p}^{*}$. In addition, white bar means final collective behaviour $\mathrm{p}^{*}$ become 1.0 and grey bar means final collective behaviour $\mathrm{p}^{*}$ become 0.01 . The number means trials lead to 1.0 . We found that when theta is between 0.1 and 0.5 , there are chances that all agents choose $\mathrm{S} 1$; it is rare.

Figure 4 Collective behaviour in scale free network, (a) final collective behaviour (b) the histogram of final collective behaviour: white bar: $\mathrm{p}^{*}=1.0$, grey bar: $\mathrm{p}^{*}=0.01$, number: trials lead to 1.0

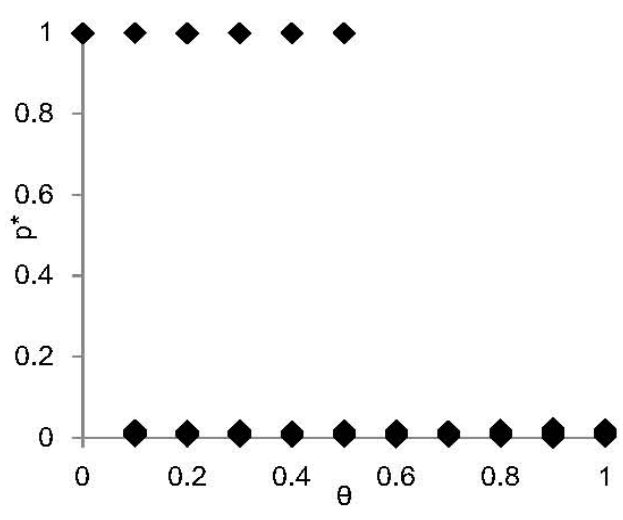

(a)

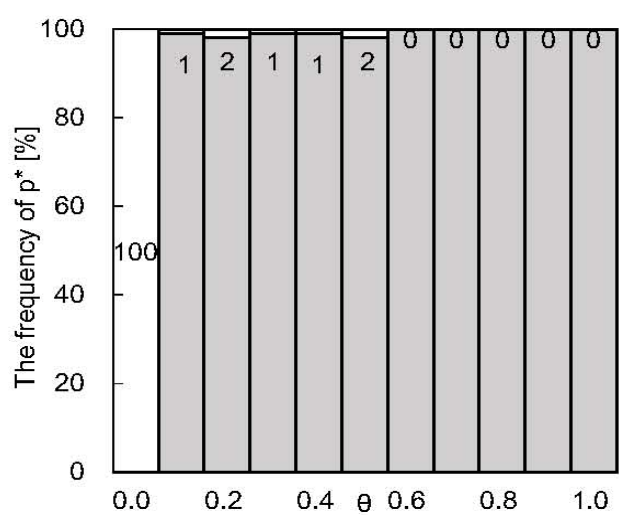

(b)

On the other hands, we show the simulation result in other social networks in Figure 5. The collective behaviour becomes 0.01 or 1.0 with depending on the trials, thresholds, which is similar to scale free network. In regular network, the range that collective behaviour becomes 0.01 or 1.0 depending on the trials is narrower than scale free network and the range is between 0.1 and 0.2 as shown in Figure 5(a). In random network, the range that collective behaviour becomes 0.01 or 1.0 depending on the trials is border than scale free network and the range is between 0.1 and 0.9 as shown in Figure 5(b). In small world network, the collective behaviour are becomes always 0.01 when theta is 0.0 and becomes always 1.0 when theta is between 0.1 and 1.0 as shown in Figure 5(c). Small world network shows unique properties with comparing other networks. I will analyse this properties in future works.

Morris (2000) deals with m-dimension lattice and shows that contagion of the action occurs below contagion threshold. In a homogeneous network, where all nodes have the same connectivity, the contagion threshold equals the inverse of the connectivity $k$. In this paper, $k$ is 10 , then contagion threshold suit for 0.1 in regular network. It is approximate for our simulation results. In our simulation results of regular network contagion threshold is 0.2 .

Moreover, López-Pintado by mean field approach showed that contagion threshold of homogeneous network is low than that of scale free network. When the degree is 10 , contagion threshold in regular network is about 0.08 and that in scale free network is 0.2 . They are approximate for our simulation results. In our simulation results of regular network contagion threshold is 0.2 and that of scale free network is 0.5 and that in random network is 0.9 as shown in Table 3. 
We found that contagion threshold depend on clustering coefficient. If clustering coefficient is large, which means that many neighbours are linked each other, contagion threshold is low and contagion is difficult to occur.

Table 3 Contagion threshold and clustering coefficient

\begin{tabular}{lcccc}
\hline & $\begin{array}{c}\text { Small } \\
\text { world }\end{array}$ & Regular & $\begin{array}{c}\text { Scale } \\
\text { free }\end{array}$ & Random \\
\hline Contagion threshold & 0.0 & 0.2 & 0.5 & 0.9 \\
Standardised clustering coefficient C/C0 & 0.99 & 1.00 & 0.11 & 0.15 \\
\hline
\end{tabular}

Figure 5 The histogram of final collective behaviour, (a) regular network (b) random network (c) small world network

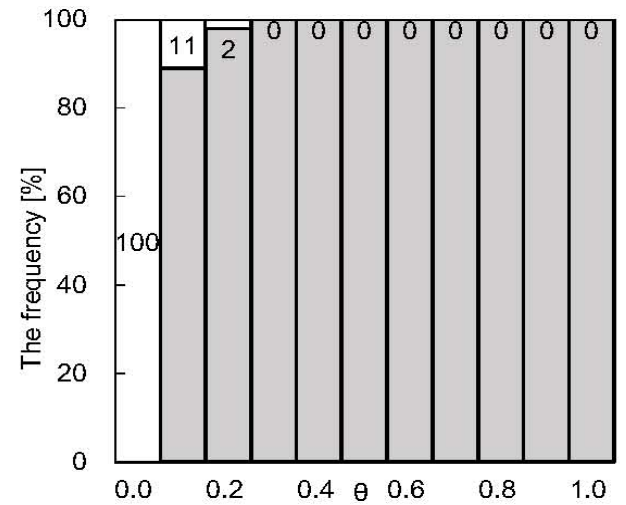

(a)

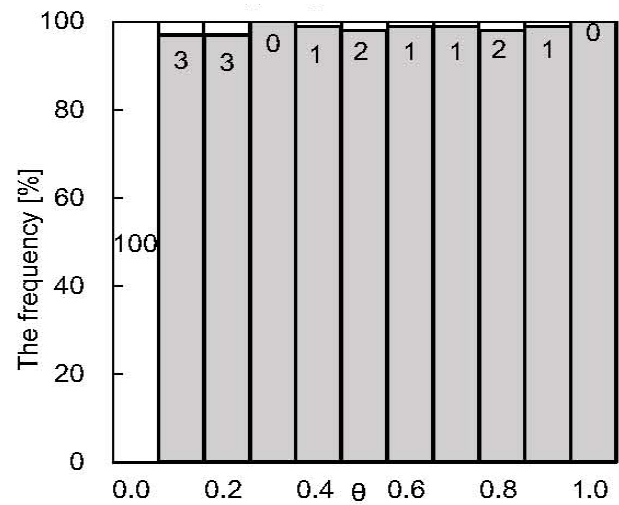

(b)

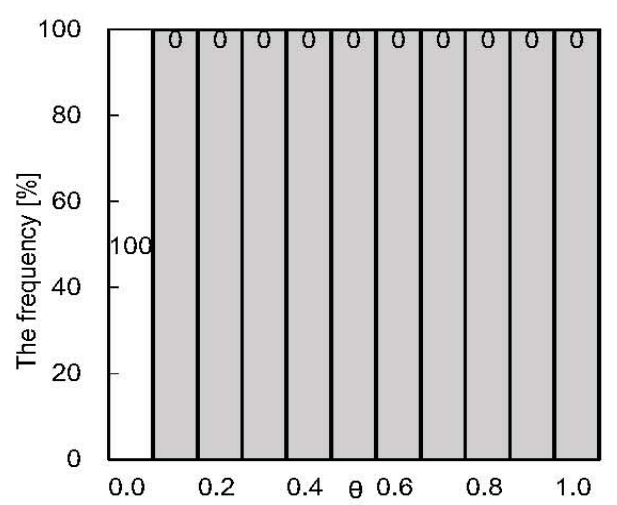

(c)

Note: White bar: $\mathrm{p}^{*}=1.0$, grey bar: $\mathrm{p}^{*}=0.01$, number: trials lead to 1.0 .

\subsection{Threshold model}

In threshold model, we found that collective behaviour depends on the initial behaviour of high degree agents (Iwanaga and Namatame, 2013). With comparing both models, we 
analyse the affection on collective behaviour in cascade model. For comparison, we show the simulation results of threshold model in Figure 6. Similar to cascade model, we made regular in which there are network 1,000 agents with ten links, and arrange the network for small world network, random network and scale free network using Kawachi algorism Then, their average degrees are 10 . We set the initial collective behaviour as 0.01 , which means only $1 \%$ of agents choose $\mathrm{S} 1$ at first time step. We set theta $\theta$ at intervals of 0.1 from 0.0 to 1.0 . We simulate until 100 time step, we call this as a trial. Then, we simulate 100 trials per each theta $\theta$ and investigate the final collective behaviour.

Figure 6 The histogram of final collective behaviour, (a) scale free network (b) regular network (c) random network (d) small world network

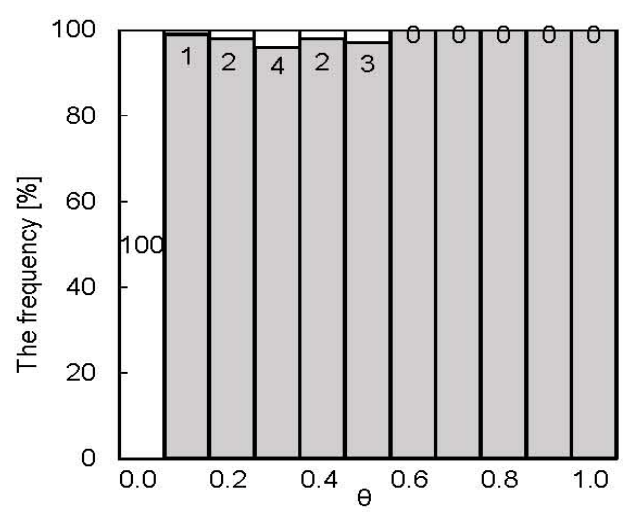

(a)

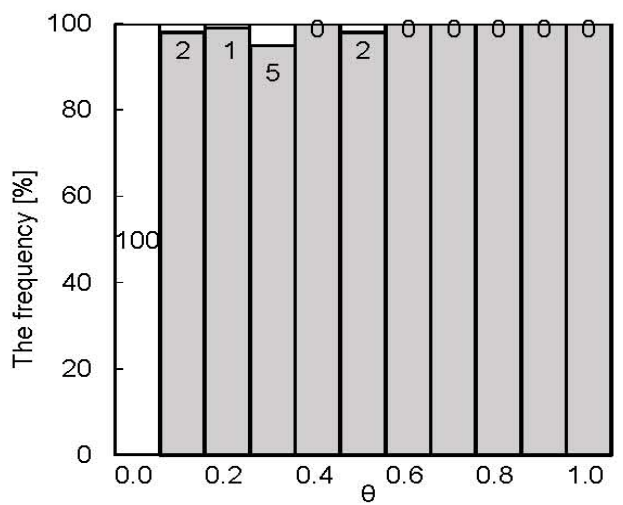

(c)

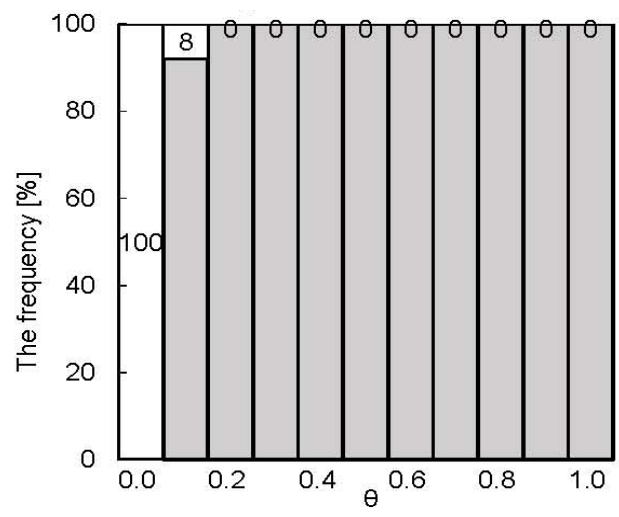

(b)

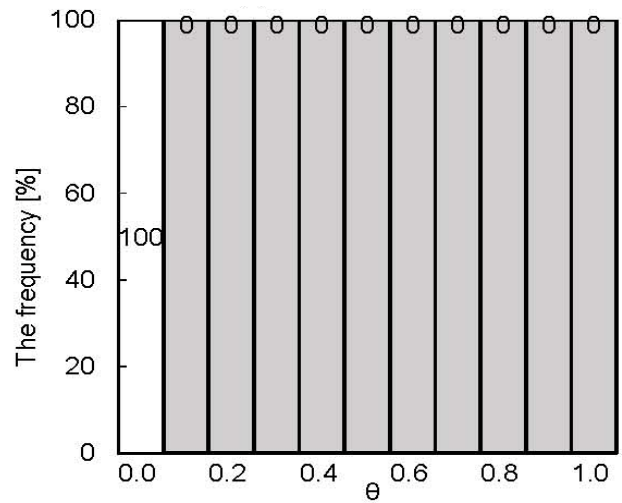

(d)

Note: White bar: $\mathrm{p}^{*}=1.0$, grey bar: $\mathrm{p}^{*}=0.0$, number: trials lead to 1.0 .

In threshold model, an agent can change from S1 to S2, or from S2 to S1. Moreover, each agent decides his behaviour depend on the neighbours' behaviour in previous time step for each time step, that is simultaneously. 
Final collective behaviour is almost same as cascade model, though the decision is interactive and simultaneously. And we showed that collective behaviour is affected in the structure of the social network and theta. Then, we found that the collective behaviour in threshold model is similar to cascade model. That is, our former results with heterogeneous rules or heterogeneous networks are possible to apply for cascade model. In these populations, each agent has an idiosyncratic threshold or networks in which agents have different numbers of neighbours. And in cascade model, it takes 10,000 times steps for a trial, but, in threshold model, it takes only 100 times steps. That means that threshold model can save the simulation resources instead of cascade model.

Note that final collective behaviour of threshold model can be 0.0 , which is shown by grey bar, because agent can change choice from S1 to S2.

We found that collective behaviour is affected in the structure of the social network and theta and the collective behaviour was stochastic. Moreover, collective behaviour is almost same as threshold model, though the decision is not interactive and simultaneously. We found that the collective behaviour in threshold model is similar to cascade model.

\section{Where is stochastic from}

\subsection{Initial decision depend on network degree}

In former section, we found that the collective behaviour of population is stochastic, although decisions of agents are deterministic. We analyse this point especially focusing scale free network. Because the differences of degree among agents in scale free network is distinct and it is easy to understand the differences. In previous simulation, agents decide at random at initial time step and each time step. Because there are agents with each degree in scale free network, we deal with the effect of network degree. We set agents' initial behaviour as ascending order or descending order with depending on degree. That is, in ascending order, agent with low degree chooses S1 preferentially at initial time step. In descending order, agent with high degree chooses S1 preferentially at initial time step.

We show the simulation results in Figure 6. The characteristic of collective behaviour is similar to the former results. We found that first decision is not effect on collective behaviour.

\subsection{Turns depend on network degree}

We set agents' turn of choice as ascending order or descending order with depending on degree. That is, in ascending order, agent with low degree decides S1 preferentially each time step. In descending order, agent with high degree decides S1 preferentially each time step.

We show the simulation results in Figure 7. The characteristic of collective behaviour is different from the former results. We found that turn of decision is effect on collective behaviour. 
Figure 7 Collective behaviour about initial decision in scale free network, (a) ascending order of network degree at initial step: white bar: $\mathrm{p}^{*}=1.0$, grey bar: $\mathrm{p}^{*}=0.01$, number: trials lead to 1.0 (b) descending order of network degree at initial step: white bar: $\mathrm{p}^{*}=1.0$, grey bar: $\mathrm{p}^{*}=0.01$, number: trials lead to 1.0

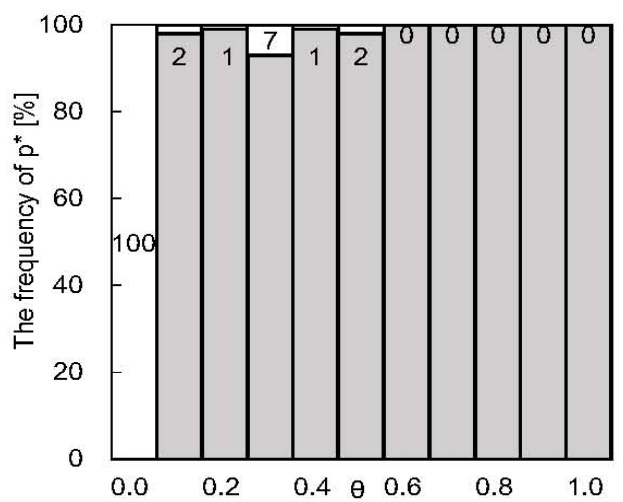

(a)

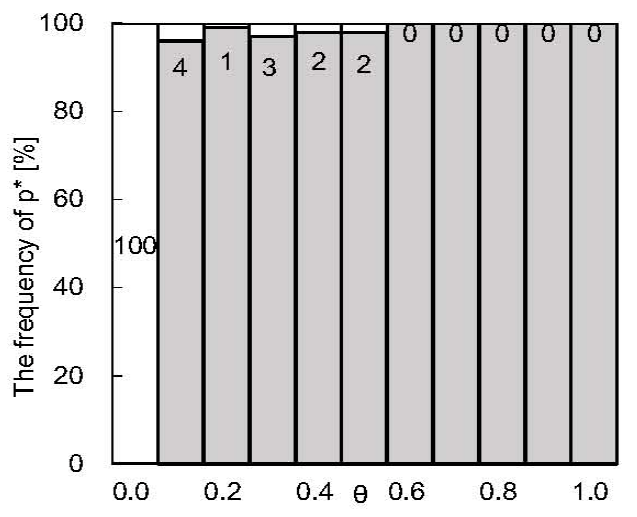

(b)

Figure 8 Collective behaviour about turns of choice in scale free network, (a) ascending order of network degree: white bar: $\mathrm{p}^{*}=1.0$, grey bar: $\mathrm{p}^{*}=0.01$, number: trials lead to 1.0 (b) descending order of network degree: white bar: $\mathrm{p}^{*}=1.0$, grey bar: $\mathrm{p}^{*}=0.01$, number: trials lead to 1.0

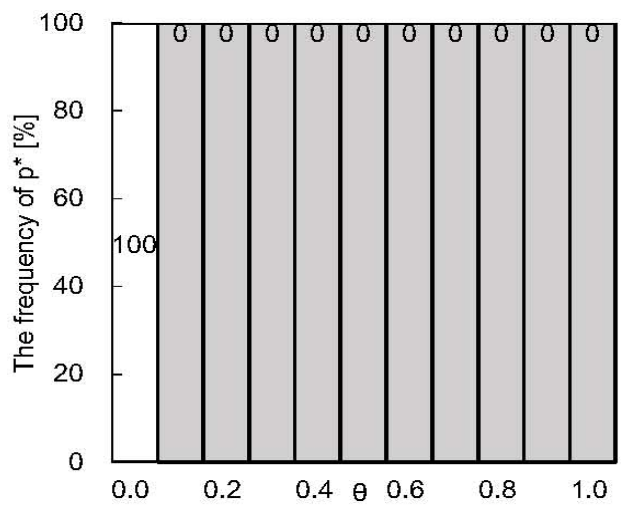

(a)

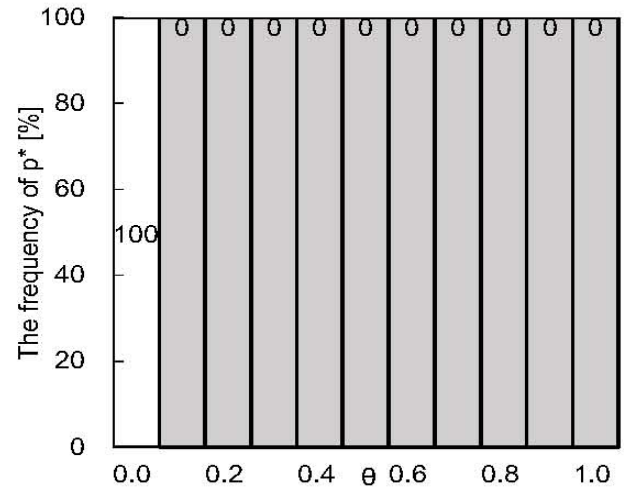

(b)

Hasan and Ukkusuri (2011) investigate effect of the initial seed on cascade propagation for uniform degree distribution. And they show that the local neighbourhood will be relatively smaller than the affected cluster size which makes the cascade to propagate fast.

We also investigated the speed of contagion and show the results in Figure 9. There are four case of contagion, ascending order of network degree at initial time step and network degree each time step, and descending order of network degree at initial time step and network degree each time step. In the first and the third case, that is bold line and bold dot line, the transitions are almost same as Figure 3. And the speed of contagion is not effected the initial seed in scale free network. On the other hand, in the second and 
the fourth case, that is solid line and dot line, we found obviously effect of turn of the choice on contagion speed in scale free network. Then, we found that turns of the choice effect on the contagion speed than initial seed in scale free network.

Here, in real world, an agent with high degree means person who has many friends to interact or communicate. Contrariwise, an agent with low degree means a person who has a few friends to. And descending order of network degree each step means that a person who has many friend choose each time step. Ascending order of network degree each step means that a person who has a few friend choose each time step.

Figure 9 Contagion speed of four cases, (a) bold line: ascending order of network degree at initial step, slid line: ascending order of network degree each step (b) bold dot line: descending order of network degree at initial step, dot line: descending order of network degree each step

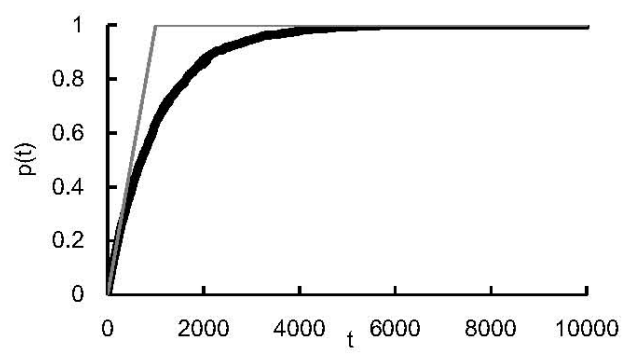

(a)

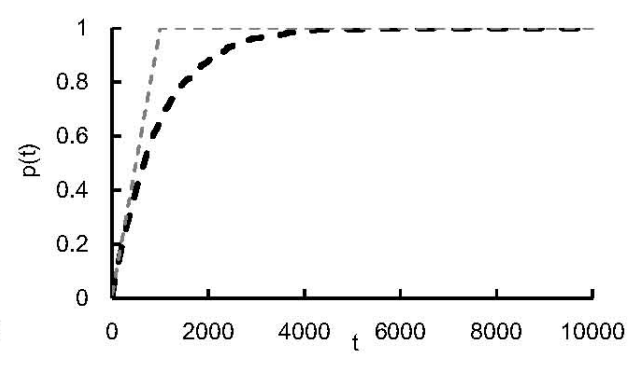

(b)

\section{Conclusions}

In cascade model, collective behaviour is affected in the structure of the social network and threshold to make decision, and the collective behaviour was stochastic, similar to threshold model in local interaction. In both models, although decisions of agents are deterministic, collective behaviour of population become stochastic. In this paper, we analysed origin of stochastic collective behaviour in cascade model. We analysed why collective behaviour of population is stochastic in scale free network. And we investigate the effect of network degree on collective behaviour. We show that turn of the choice effects on collective behaviour not the first choices. And we found that collective behaviour is affected by turns of decisions not the first choices. Moreover, contagion speed is also affected by turns of decisions than initial decisions.

\section{References}

Albert, R. and Barabási, A.L. (2000) 'Topology of evolving networks: local events and universality', Physical Review Letters, Vol. 85, No. 24, pp.5234-5237.

Arthur, W.B. (1989) 'Competing technologies, increasing returns, and lock-in by historical events', The Economic Journal, Vol. 99, No. 394, pp.116-131.

Arthur, W.B. (1994) 'Inductive reasoning and bounded rationality', American Economic Review, Vol. 84, No. 2, pp.406-411.

Axelrod, R. (1984) The Evolution of Cooperation, Basic Books, New York. 
Feng, L., Li, B., Podobnik, B., Preis, T. and Stanley, H.E. (2012) 'Linking agent-based models and stochastic models of financial markets', The National Academy of Sciences of the United States of America (PNAS), Vol. 109, No. 22, pp.8388-8393.

Fudenberg, D. and Levine, D. (1998) The Theory of Learning in Games, The MIT Press, Cambridge.

Hansarnyi, J. and Selten, R. (1988) A Game Theory of Equilibrium Selection in Games, MIT Press, Cambridge.

Hasan, S. and Ukkusuri, S.V. (2011) 'A threshold model of social contagion process for evacuation decision making', Transportation Research Part B: Methodological, Vol. 45, No. 10, pp.1590-1605.

Huberman, B.A. and Glance, N.S. (1993) 'Diversity and collective action: interdisciplinary approaches to nonlinear complex systems', Springer Series in Synergetics, Vol. 62, pp.44-64

Iwanaga, S. and Namatame, A. (2012) 'Collective behavior and diverse social network', International Journal of Advancements in Computing Technology, Vol. 4, No. 22, pp.321-320.

Iwanaga, S. and Namatame, A. (2013) 'Collective behavior in cascade and Schelling model', Procedia Computer Science: 17th Asia Pacific Symposium on Intelligent and Evolutionary Systems (IES2013), Vol. 24, pp.217-226.

Kawachi, Y., Murata, K., Yoshii, S. and Kakazu, Y. (2004) 'The structural phase transition among fixed cardinal networks', Proceedings of the 7th Asia-Pacific Complex Systems Conference, Cairns, Australia, pp.247-255.

Kenett, D.Y., Raddant, M., Lux, T. and Ben-Jacob, E. (2012) 'Evolvement of uniformity and volatility in the stressed global financial village', Public Library of Science: PLoS ONE [online] http://journals.plos.org/plosone/article?id=10.1371/journal.pone.0031144 (accessed 26 January 2015).

Kirman, A.P. (1997) 'The economy as an interactive system', in Arthur, W.B., Durlauf, S.N. and Lane, D. (Eds.): The Economy as an Evolving Complex System II, Perseus, New York.

Komatsu, T. and Namatame, A. (2012) Evolutionary Optimized Network Topology for Maximizing Cascade, The Special Interest Group Technical Reports of Processing Society of Japan (IPSJ SIG Technical Report), Vol. 2012-ICS-166, No. 3, pp.1-6.

López-Pintado, D. (2006) 'Contagion and coordination in random networks', International Journal of Game Theory, Vol. 34, No. 3, pp.371-381.

Montanari, A. and Saberi, A. (2010) 'The spread of innovation in social networks', The National Academy of Sciences of the United States of America (PNAS), Vol. 107, No. 47, pp.20196-20201.

Morris, S. (2000) 'Contagion', Review of Economic Studies, Vol. 67, No. 1, pp.57-78.

Nowak, M.A. (2006) Evolutionary Dynamics: Exploring the Equations of Life, Belknap Press, Cambridge.

Rubinstein, A. (1998) Modeling Bounded Rationality, The MIT Press, Cambridge.

Schelling, T. (1978) Micromotives and Macrobehavior, Norton, New York.

Schweitzer, F. (2002) 'Brownian agent models for swarm and chemotactic interaction', Proceedings Fifth German Workshop on Artificial Life. Abstracting and Synthesizing the Principles of Living Systems, pp.181-190.

Sipper, M. (1997) Evolution of Parallel Cellular Machines: The Cellular Programming Approach, Springer-Verlag, Heidelberg.

Watts, D.J. (1999) Small-Worlds: The Dynamics of Networks between Order and Randomness, Princeton University Press, Princeton.

Watts, D.J. (2002) 'A simple model of global cascades on random networks', The National Academy of Sciences of the United States of America (PNAS), Vol. 99, No. 9, pp.5766-5771.

Young, H.P. (2011) 'The dynamics of social innovation', The National Academy of Sciences of the United States of America (PNAS), Vol. 108, No. 4, pp.21285-21291. 